\title{
ANÁLISIS DE FACTORES DE RIESGO ASOCIADOS CON ANOMALÍAS CONGÉNITAS EN RECIÉN NACIDOS DE LAZONA DE LA CUENCA DEL RÍO MATANZA-RIACHUELO.
}

\section{ANALYSIS ON RISK FACTORS ASSOCIATED WITH BIRTH DEFECTS IN NEWBORNS IN THE AREA OF THE MATANZA-RIACHUELO RIVER BASIN.}

\author{
María Paz Bidondo ${ }^{1}$, Boris Groisman ${ }^{1,6}$, Juan Antonio Gili ${ }^{2}$, Rosa Liascovich ${ }^{1}$, María Cecilia Martin ${ }^{1}$, Ana \\ Tocci $^{3}$, Romina Flores ${ }^{4}$, Andrea Pus Barraza ${ }^{5}$, Pablo Barbero ${ }^{1}$ \\ 1 Red Nacional de Anomalías Congénitas (RENAC), Centro Nacional de Genética Médica, ANLIS "Carlos Malbrán". \\ 2 Estudio Colaborativo Latinoamericano de Malformaciones Congénitas (ECLAMC) en Laboratorio de Epidemiología Genética, Dirección de Investigación CEMIC- \\ CONICET, Buenos Aires. \\ 3 Hospital General de Agudos “Dr. Cosme Argerich”, Ciudad de Buenos Aires. \\ 4 Hospital Interzonal General de Agudos "Evita", Lanús, provincia de Buenos Aires. \\ 5 Hospital Zonal General de Agudos "Dr. Diego Paroissien", La Matanza, provincia de Buenos Aires. \\ 6 Email de contacto: bgroisman@gmail.com
}

\section{Conceptos clave}

A) Qué se sabe sobre el tema:

Existen investigaciones sobre la asociación entre población expuesta a posibles fuentes de contaminación ambientales (fábricas, basurales, etc.) y ocurrencia de anomalías congénitas. Sin embargo la evidencia aún es controversial, no siendo los resultados claramente consistentes.

B) Qué aporta este trabajo:

Se trata de la primera investigación sobre factores de riesgo de anomalías congénitas con datos georreferenciados en el área de la cuenca Matanza Riachuelo, un área ampliamente contaminada, mostrando la factibilidad de efectuar este tipo de estudios

\section{Resumen:}

Introducción: en la cuenca Matanza-Riachuelo viven aproximadamente 4.885 .000 habitantes y es uno de los sitios más contaminados de Argentina. Este estudio evaluó factores de riesgo asociados a anomalías congénitas (AC) y bajo peso al nacimiento. Métodos: se realizó un estudio caso-control en tres hospitales de la cuenca pertenecientes a la Red Nacional de Anomalías Congénitas (RENAC) durante un año. Se evaluó asociación de bajo peso y AC, con variables socioeconómicas; reproductivas; distancia de la vivienda durante la gestación a industrias y a cursos de agua. Resultados: la prevalencia de anomalías congénitas en recién nacidos fue de 1,93\% (IC 95\%: 1,64 - 2,25). Los factores de riesgo asociados a AC fueron enfermedades maternas agudas y consumo de medicamentos. Los casos presentaron peso y edad gestacional significativamente más bajos y mayor edad materna. No se encontró asociación entre bajo peso o prevalencia de AC con distancia de la vivienda durante el embarazo a industrias ni a los cursos de agua. Conclusión principal: este estudio es la primera investigación en el área de las $A C$ con datos georreferenciados en nuestro país y ha mostrado la factibilidad de efectuar este tipo de investigaciones para evaluar factores de riesgo en la RENAC. Futuras investigaciones orientadas a evaluar AC específicas deberían realizarse a fin de corroborar los resultados del presente estudio.

Palabras clave: anomalías congénitas; contaminantes del aire; factores de riesgo; Argentina.

\section{Abstract:}

Introduction: Matanza-Riachuelo basin is one of the most polluted sites in Argentina, with 4,885,000 inhabitants. This study evaluated risk factors associated with congenital anomalies (CA) and low birth weight. Methods: case-control study in three hospitals belonging to the National Network of Congenital Anomalies (RENAC) for one year. We evaluated the association of socioeconomic level, reproductive factors, and distance between place of residence to industrial pollution places and from watercourses of the river, with low birth weight and CA. Results: The prevalence of congenital anomalies in newborn was $1.93 \%$ (95\% Cl:1.64 - 2.25). There were associated risk factors such as acute maternal diseases and consumption of drugs. Cases had weight and gestational age significantly lower and higher maternal age than the controls. We did not find association between low birth weight and prevalence of congenital anomalies with distance from the housing of pregnant women to sources of contamination. Conclusions: This study is the first research in Argentina with geo-referenced data and newborn with CA. It has shown the feasibility of carrying out this type of research to evaluate risk factors in the RENAC. Future research to assess specific type of CA should be conducted to corroborate the results of this study.

Keywords: congenital abnormalities; air pollutants; risk factors; Argentina. 


\section{Introducción}

Las anomalías congénitas (AC) son actualmente en Argentina la segunda causa de mortalidad infantil y explican aproximadamente un $28 \%$ de las defunciones en menores de 1 año ${ }^{1}$. Asimismo, las AC ocasionan un gran impacto en la morbilidad pues implican trastornos graves, frecuentemente crónicos, discapacitantes y con un alto impacto para los afectados, sus familias y el sistema de salud. Dentro de las causas de $\mathrm{AC}$ aquellos casos asociados a factores ambientales (teratogénicos) son evitables mediante la aplicación de medidas de prevención primaria. La cuenca Matanza-Riachuelo (CMR) abarca un área de $2.240 \mathrm{~km} 2$ y está integrada por 14 partidos del conurbano bonaerense y la zona sur de la Ciudad Autónoma de Buenos Aires (CABA). La CMR es considerada uno de los sitios más contaminados del mundo, siendo las principales fuentes de polución el vertido de efluentes industriales y de líquidos cloacales, los basurales a cielo abierto y el polo petroquímico de Dock Sud. La carga contaminante que se vierte en las aguas de la cuenca supera ampliamente su capacidad diluyente y de autodepuración ${ }^{2,3}$. Diferentes estudios en otros ámbitos han investigado la asociación entre población expuesta a posibles fuentes de contaminación ambientales (fábricas, basurales, etc.) y ocurrencia de anomalías congénitas, no siendo los resultados claramente consistentes ${ }^{4}$. Considerando los múltiples factores de riesgo a los que está expuesta la población que vive en la CMR y la ausencia de investigaciones orientadas a identificar un potencial efecto teratogénico, se consideró necesario desarrollar un estudio de investigación con el objetivo de evaluar factores de riesgo asociados a la ocurrencia de anomalías congénitas, y de recién nacidos con bajo peso en hospitales seleccionados de la Red Nacional de Anomalías Congénitas localizados en la zona de la cuenca Matanza-Riachuelo.

\section{Métodos}

Se realizó un estudio observacional analítico de casos y controles que incluyó todos los nacimientos ocurridos durante el periodo del 1ro. de junio 2012 al 31 de mayo del 2013 en los hospitales Argerich de la CABA, Paroissien del partido de la Matanza y Evita del partido de Lanús. Para el presente estudio se incluyeron el total de recién nacidos vivos, y los fetos muertos de 500 gramos o más con AC estructurales mayores detectadas hasta el alta/egreso del hospital. Por cada recién nacido vivo con AC (casos) se tomaron 4 controles del mismo sexo y nacidos en el mismo hospital que el caso. Para el estudio de la asociación entre las variables de exposición y AC se incluyó el total de casos y el total de controles. Para el estudio de la asociación entre las variables de exposición y el bajo peso al nacer, no se consideraron los recién nacidos con $\mathrm{AC}$ sino que al total de controles se lo dividió entre los recién nacidos con peso menor a 2.500 gramos y aquellos con un peso mayor. Los hospitales participantes forman parte de la RENAC y la descripción de los pacientes con anomalías siguió las normas operativas de dicha red.

Los datos de casos y controles se recolectaron usando un formulario especial. En cada hospital participante, el profesional a cargo del proyecto realizó el interrogatorio de la madre y el examen del recién nacido, tanto en los casos como en los controles. Se incluyeron todas las AC que presentó cada caso. En la coordinación de la RENAC se realizó la codificación de las AC según la Clasificación Internacional de Enfermedades, Décima Revisión con la adaptación de la Colegio Real de Pediatría y Salud Infantil (ICD10-RCPCH, 2011). El domicilio de residencia de la madre durante la gestación fue georreferenciado con latitud y longitud. Para localizar las fuentes de contaminación se utilizó la información georreferenciada de las industrias por rubro y de los cursos de agua de la $\mathrm{CMR}^{\text {(Figuras } 1 \text { y 2) }}$. 
Figura 1. Distribución geaográfica de las industrias (A) y cursos de agua y hospitales (B); en la región de la cuenca Matanza Riachuelo.

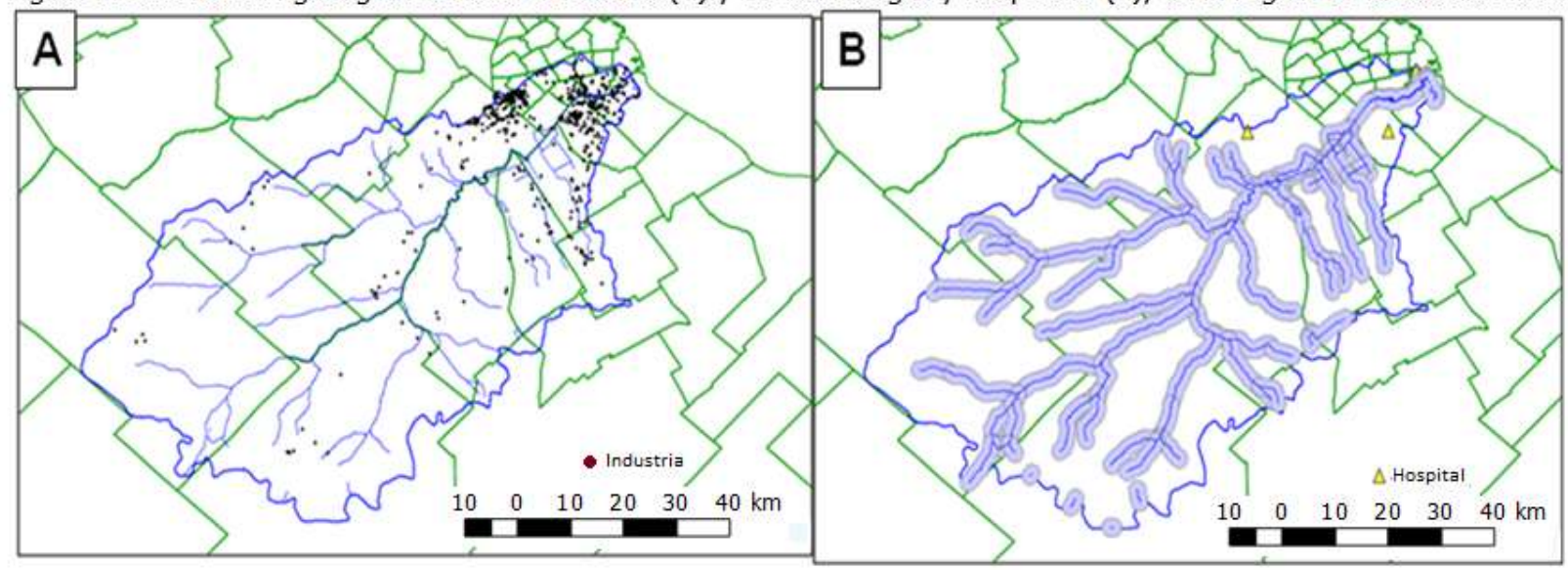

Figura 2. Lugar de residencia durante el embarazo de casos (A) y controles (B); en la región de la cuenca Matanza Riachuelo; hospitales Argerich, Paroissien y Evita período01/06/12 - 31/05/13.

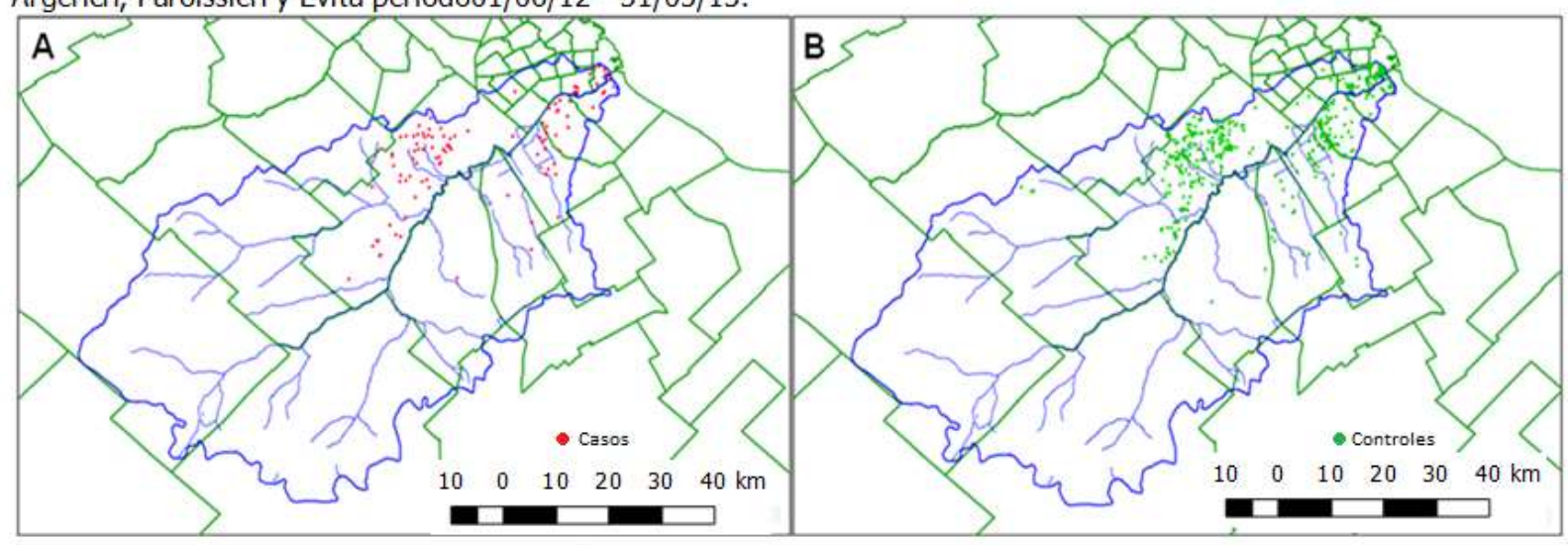

Se analizaron las siguientes variables: edad gestacional, peso de los recién nacidos, paridad (primíparas vs multíparas), gemelaridad, nivel de instrucción materno y paterno (hasta primaria completa vs secundaria incompleta o más), nivel de ocupación materno y paterno (ocupado/estudiante vs desocupada), enfermedad aguda materna (presencia o no), enfermedad crónica materna (presencia o no), consumo de medicamentos (expuestas vs no expuestas) consumo de tabaco (expuestas vs no expuestas), consumo de alcohol (expuestas vs no expuestas), consumo de drogas de abuso (expuestas vs no expuestas), tipo de vivienda (casa/departamento vs otro tipo de vivienda inquilinato/rancho/casilla), fuente de agua utilizada (agua de red vs perforación/pozo), tratamiento del agua (tratada -hervir/filtrar- vs sin tratamiento), desagüe cloacal (red pública -cloaca- vs sin red pública -pozo ciego/cámara séptica). En una segunda instancia se realizó un análisis con las mismas variables sólo en la población de recién nacidos sanos (controles), comparando los que tenían bajo peso (<2.500 gramos) con los recién nacidos que no tenían bajo peso. Se compararon las proporciones a través de la prueba de chi cuadrado y las variables continuas con test de t. Se definió un nivel de significación $p<0,05$.

El estudio de asociación entre la distancia de la vivienda de la mujer embarazada a focos de contaminación industrial y a los cursos de agua de la cuenca también se realizó en casos y controles; en una segunda instancia se realizó un análisis con las mismas variables sólo en la población de recién nacidos sanos (controles) comparando los que tenían bajo peso con los recién nacidos que no tenían bajo peso. Para este análisis sólo se incluyeron los casos y controles residentes dentro de la CMR: 107 casos y 443 controles respectivamente. Se excluyeron 52 casos y 157 controles residentes fuera de la región de la CMR. Para este análisis se consideraron las principales industrias de la CMR por rubro (tabla 1) y su geolocalización (latitud y longitud), así como la geolocalización de los cursos de agua. 
Tabla 1. Industrias según rubro en la cuenca Matanza Riachuelo

\begin{tabular}{|c|c|c|}
\hline \multirow{2}{*}{ Rubro } & \multicolumn{2}{|c|}{ Industrias por rubro } \\
\cline { 2 - 3 } & $\mathrm{N}$ & 44,64 \\
\hline Alimenticia & 208 & 18,24 \\
\hline Curtiembre & 85 & 13,30 \\
\hline Metalurgia & 62 & 3,65 \\
\hline Papelera & 17 & 13,52 \\
\hline Química & 63 & 6,65 \\
\hline Textil & 31 & 100,00 \\
\hline Total & 466 & \\
\hline
\end{tabular}

Para cada caso y control se efectuó la geolocalización. Este análisis se realizó con tres indicadores de distancia a las industrias y al curso de agua:

1. Suma de las distancias: para cada caso y para cada control se calcularon las distancias a todas las industrias de un rubro y a los cursos de agua. Luego se efectuó la suma de todas las distancias y se dividió por el total de individuos tanto para casos como para controles.

2. Media de las distancias: para cada caso y para cada control se calculó la distancia media a todas las industrias de un rubro y a los cursos de agua. Luego se calculó el promedio de las medias tanto para casos como para controles.

3. Distancia mínima: para cada caso y para cada control se calculó la distancia a la industria por rubro y curso de agua más cercano. Luego se calculó el promedio de dichas distancias tanto para los casos como para los controles. Para cada valor se estimó el promedio, desvío estándar y error estándar. Los promedios se compararon con el test de t. Para el estudio de asociación con distancia de la vivienda de la mujer embarazada a los cursos de agua de la cuenca se hizo además un análisis dicotómico según si la vivienda estaba a una distancia menor a 1.000 metros de los cursos de agua o mayor a esa distancia, y en una segunda instancia se dividieron las distancias en 5 quintilos y se compararon el primer y quinto quintilo. Se compararon las proporciones a través de la prueba de chi cuadrado. Se definió un nivel de significación $\mathrm{p}<0,05$.

Finalmente se realizó un análisis de distribución espacial de individuos con el objetivo de verificar si los casos estaban localizados en áreas con alta concentración respecto a los controles siguiendo un modelo de distribución de Bernoulli. Se utilizó el software SatScan v9.1.1 (www.satscan.org). Se definió un nivel de significación $p<0,05$.

\section{Resultados}

El total de recién nacidos vivos evaluado fue de 8.255 de los cuales 160 presentaron anomalías congénitas resultando una prevalencia de 1.93\% (IC 95\%: 1,64 - 2,25).

Entre los factores de exposición durante el embarazo sólo se observó una mayor frecuencia de enfermedades maternas agudas en los casos (Tabla 2). No se observaron diferencias significativas en las variables socioeconómicas, ni en las variables habitacionales ni en las características reproductivas entre casos y controles (Tabla 2). Los casos presentaron una media de edad materna de 27,5 años, lo que resultó significativamente mayor que la de los controles, que fue de 25,4 años ( $p<0,001$ ); la edad gestacional media de los casos resultó significativamente más baja, de 37,1 semanas, que la de los controles, que fue de 38,7 ( $p<0,001$ ). El peso medio de los casos fue de 2.811 gramos lo que resultó significativamente más bajo que los controles, que fue de 3.243 gramos $(p<0,001)$.

De los 600 controles, $71(11,83 \%)$ presentaron bajo peso (<2.500 gramos). Entre los controles con bajo peso y aquellos con peso adecuado, sólo se observaron diferencias significativas en la proporción de gemelaridad (Tabla 2). 
Tabla 2. Características reproductivas, socioeconómicas y factores de exposiciones durante el embarazo en casos vs controles; bajo peso vs peso adecuado; hospitales Argerich, Paroissien y Evita.

Período 01/06/12 - 31/05/13

\begin{tabular}{|c|c|c|c|c|c|c|}
\hline & \multicolumn{3}{|c|}{ Caso-control } & \multicolumn{3}{|c|}{ Solo controles } \\
\hline Variable & $\begin{array}{l}\text { Casos } \\
\text { N (\%) }\end{array}$ & $\begin{array}{l}\text { Controles } \\
\text { N (\%) }\end{array}$ & $p$ & $\begin{array}{l}\text { Bajo peso } \\
\text { N }(\%)\end{array}$ & $\begin{array}{r}\text { Peso } \\
\text { adecuado } \\
(\%)\end{array}$ & $p$ \\
\hline Primiparidad & $41(25,62)$ & $181(30,15)$ & 0,263 & $28(39,44)$ & $153(28,92)$ & 0,076 \\
\hline Gemelaridad & $6(3,75)$ & $31(5,20)$ & 0,497 & $16(22,53)$ & $15(2,83)$ & $0,001 *$ \\
\hline Desocupación materna & $104(65,00)$ & $398(66,33)$ & 0,875 & $41(57,75)$ & $357(67,48)$ & 0,115 \\
\hline Desocupación paterna & $17(11,00)$ & $40(6,66)$ & 0,079 & $2(2,81)$ & $38(7,18)$ & 0,185 \\
\hline $\begin{array}{l}\text { Instrucción materna hasta 1ria } \\
\text { completa }\end{array}$ & $46(28,75)$ & $184(30,66)$ & 0,932 & $23(32,39)$ & $161(30,43)$ & 0,735 \\
\hline $\begin{array}{l}\text { Instrucción paterna hasta 1ria } \\
\text { completa }\end{array}$ & $93(58,12)$ & $354(59,00)$ & 0,48 & $38(53.52)$ & $316(59,73)$ & 0,531 \\
\hline Vivienda precaria & $22(13,75)$ & $84(14,00)$ & 0,701 & $11(15,5)$ & $73(13,80)$ & 0,666 \\
\hline Sin agua de red & $10(6,25)$ & $61(10,16)$ & 0,701 & $9(12,67)$ & $52(9,82)$ & 0,456 \\
\hline Sin desague claocal & $78(48,75)$ & $318(53,00)$ & 0,828 & $40(56,33)$ & $278(52,55)$ & 0,587 \\
\hline Enfermedad aguda & $78(48,75)$ & $274(45,66)$ & $0,048 *$ & $34(47,88)$ & $240(45,36)$ & 0,643 \\
\hline Enfermedad crónica & $24(15,00)$ & $78(13,00)$ & 0,247 & $11(15,49)$ & $67(12,66)$ & 0,448 \\
\hline Medicamentos & $96(60,00)$ & $331(55,16)$ & $0,016^{*}$ & $40(56,34)$ & $291(55,00)$ & 0,788 \\
\hline Tabaquismo & $26(16,25)$ & $118(19,66)$ & 0,556 & $16(22,53)$ & $102(19,28)$ & 0,527 \\
\hline Alcohol & $15(9,37)$ & $62(10,33)$ & 0,842 & $10(14,08)$ & $52(9,82)$ & 0,527 \\
\hline Radiación ionizante & $8(5,00)$ & $17(2,83)$ & 0,095 & $3(4,22)$ & $14(2,64)$ & 0,463 \\
\hline Drogas de abuso & $1(0,62)$ & $6(1,00)$ & 0,75 & $2(2,81)$ & $4(0,75)$ & 0,102 \\
\hline
\end{tabular}

En el análisis de asociación entre la distancia de la vivienda de la mujer embarazada a focos de contaminación industrial no se detectaron diferencias significativas tanto en la comparación de casos y controles, como en el análisis de los recién nacidos con peso adecuado y con bajo peso, con ninguna de las tres mediciones de distancia utilizadas (tabla 3).

Tabla 3. Distancia entre lugar de residencia e industrias según rubro (media de distancias) de casos vs controles; bajo peso vs peso adecuado; hospitales Argerich, Paroissien y Evita período 01/06/12 - 31/05/13.

\begin{tabular}{|c|c|c|c|c|c|c|}
\hline \multirow[b]{2}{*}{$\begin{array}{c}\text { Tipo de } \\
\text { industria }\end{array}$} & \multicolumn{3}{|l|}{ Caso-control } & \multicolumn{3}{|c|}{ Solo controles } \\
\hline & \begin{tabular}{l}
\multicolumn{2}{c}{ Casos } \\
$\underset{\text { km }}{\text { media }}$ (DS)
\end{tabular} & $\begin{array}{c}\text { Controles } \\
\text { media (DS) } \\
\text { km }\end{array}$ & $p$ & $\begin{array}{l}\text { Bajo peso } \\
\text { media (DS) } \\
\quad \text { km }\end{array}$ & $\begin{array}{c}\text { Peso } \\
\text { adecuado } \\
\text { media (DS) } \\
\text { km }\end{array}$ & $p$ \\
\hline Alimenticia & $1.66(0.32)$ & $1.63(0.32)$ & 0,4355 & $1.67(0.43)$ & $1.63(0.30)$ & 0,3389 \\
\hline Curtiembres & $1.38(0.85)$ & $1.31(0.81)$ & 0,4578 & $1.31(0.93)$ & $1.31(0.79)$ & 0,5237 \\
\hline Metalúrgica & $1.38(0.63)$ & $1.33(0.59)$ & 0,4166 & $1.34(0.73)$ & $1.33(0.57)$ & 0,8439 \\
\hline Papelera & $1.35(0.67)$ & $1.35(0.63)$ & 0,3448 & $1.37(0.77)$ & $1.35(0.61)$ & 0,8392 \\
\hline Química & $1.54(0.46)$ & $1.50(0.44)$ & 0,4025 & $1.52(0.57)$ & $1.50(0.42)$ & 0,7213 \\
\hline Textil & $1.28(0.43)$ & $1.24(0.42)$ & 0,3372 & $1.29(0.55)$ & $1.23(0.40)$ & 0,3318 \\
\hline
\end{tabular}

DS: desvio estandar

La distancia media entre el lugar de residencia durante el embarazo y los cursos de agua de la cuenca no resultó significativamente diferente entre los casos y los controles (la distancia media mínima en ambos 


\section{Factores de riesgo de anomalías congénitas-}

grupos fue de $0,14 \mathrm{~km}$ ), ni al comparar recién nacidos de bajo peso con peso adecuado (distancias medias minimas de $0,15 \mathrm{~km}$ y $0,14 \mathrm{~km}$, respectivamente).

En el análisis de distribución espacial de casos y controles no se observó un agrupamiento de casos significativamente diferente a la distribución de controles.

\section{Discusión}

El presente es el primer trabajo epidemiológico orientado a analizar e investigar factores de riesgo teratogénico en una muestra de la población de la CMR.

La prevalencia de anomalías congénitas en recién nacidos vivos observada en este estudio se encuentra dentro de las prevalencias observadas en el total de la RENAC 1,76\% (IC 95\% 1,71 a 1,80) ${ }^{5}$. Las madres de los casos presentaron edades mayores y mayor proporción de multiparidad, factores que se sabe que están asociados al mayor riesgo de anomalías cromosómicas relacionado con la edad avanzada. De las otras variables analizadas se observó una mayor frecuencia de exposición a enfermedades agudas y medicamentos en los casos. La exposición a ciertas enfermedades agudas puede implicar un mayor riesgo teratogénico en especial las infecciones del grupo TORCH (Toxoplasma, Herpes, Citomegalovirus, Rubéola y Otras), así como también la exposición a medicamentos de riesgo teratogénico reconocido. La exposición a enfermedades crónicas, radiaciones ionizantes, alcohol y otras drogas de abuso son factores de riesgo reconocidos. Sin embargo, en nuestro estudio no se identificó una asociación significativa con estas variables. Esta situación quizá se deba al tamaño muestral reducido y al hecho que estos factores pueden causar anomalías que se manifiestan en edades posteriores de la vida, como por ejemplo, la discapacidad intelectual. Un aspecto a destacar es la proporción de gestantes que refirieron haber fumado $(19,5 \%)$ y consumido alcohol $(10,0 \%)$. La proporción de mujeres que refirieron fumar durante el embarazo fue cercana a las observada en la segunda encuesta nacional de factores de riesgo que mostró una frecuencia de tabaquismo en mujeres no gestantes menores de 65 años del $22,4 \%{ }^{6}$. El consumo de alcohol en nuestro estudio fue mayor al reportado previamente $(6,9 \%)$. Sin embargo estas diferencias pueden responder a una definición diferente sobre consumo de alcohol ${ }^{6}$. Dado que no hay una dosis mínima segura sobre consumo de alcohol en el embarazo resultan preocupantes los resultados observados en nuestro estudio.

La presencia de un peso al nacimiento significativamente más bajo y la edad gestacional menor en los casos es coincidente con lo ya ampliamente reportado en la literatura ${ }^{7}$.

En un estudio previo realizado en algunas maternidades de Argentina se identificó una asociación significativa entre el nivel socioeconómico bajo y un mayor riesgo de fisura de labio con/sin paladar hendido $(O R=1,43)$ y comunicación interventricular $(O R=1,38)^{8}$. Ese estudio incluyó instituciones públicas y privadas y de diferentes regiones del país. Coincidentemente en otra investigación realizada con datos provenientes de cuatro registros de Reino Unido se detectó un mayor riesgo para anomalías congénitas OR $1.33\left(95 \%\right.$ Cl 1.01 a 1.75) y nivel socioeconómico bajo ${ }^{9}$. En nuestro estudio no identificamos un mayor riesgo de $\mathrm{AC}$ y el nivel socioeconómico bajo.

La asociación entre factores habitacionales y anomalías congénitas no ha sido evaluada en estudios previos en nuestra población. Un aspecto a tener en cuenta en relación a estas variables es que si bien parte de las mujeres no cuentan con agua potable, una alta proporción realiza tratamiento del agua para beber (agua hervida). Al mismo tiempo los datos de nuestro estudio muestran que el $90 \%$ de las mujeres cuentan con conexión a la red de agua pública, cifra superior a la reportado pocos años antes, lo cual indicaría una mejora en las condiciones de vida de esta población, lo que posiblemente operó homogenizando a la muestra para esta variable.

En relación a los niños sin anomalías congénitas con bajo peso, la única variable que mostró una asociación estadísticamente significativa fue la gemelaridad, hecho ampliamente conocido. En estudios previos, el bajo peso fue asociado al nivel educativo de las madres, en especial al comparar los grados más extremos en las escalas educativas alcanzadas ${ }^{10}$. En nuestro estudio no identificamos una asociación con esta variable quizá por la homogeneidad de la muestra estudiada.

La falta de asociación entre anomalías congénitas y bajo peso en relación a la distancia a focos industriales y los cursos de agua, puede responder a la ausencia real de una asociación o bien a 
limitaciones metodológicas. Algunas investigaciones han identificado un mayor riesgo de anomalías congénitas en poblaciones que viven próximas a fuentes contaminantes ${ }^{11,12,13,14,15,16,17}$ mientras que otras no han detectado esta asociación ${ }^{18,19,20}$. La falta de consistencia entre los estudios en este campo posiblemente responda a la heterogeneidad entre las diferentes situaciones de contaminación, condiciones de vida de las poblaciones estudiadas y a las dificultades metodológicas de las investigaciones en esta área en especial la evaluación de la exposición a contaminantes ${ }^{(4)}$. En un estudio de revisión reciente sobre las investigaciones dirigidas a investigar la asociación entre factores ambientales y anomalías congénitas o bajo peso, se ha señalado la gran heterogeneidad de los estudios y las limitaciones metodológicas de los mismos ${ }^{21}$. Dadas las dificultades referidas en el presente estudio se analizó la exposición a focos industriales y cursos de agua desde diferentes metodologías. Sin embargo en ninguna de ellas se detectó una diferencia significativa. Una posible explicación pudiera estar relacionada con un lugar de residencia más alejado a los hospitales de atención de los casos en relación a los controles. Esta situación posiblemente esté relacionada con el diagnóstico prenatal de la anomalía y la derivación selectiva de estas gestantes. Sin embargo, solo uno de los hospitales participantes (Hospital Argerich) mostró una diferencia significativa en la distancia entre la residencia y el hospital donde ocurrió el nacimiento. Tampoco se identificó algún área de alta concentración de casos, lo cual es coincidente con la falta de asociación con un foco de contaminación específico.

En cuanto a las fortalezas, se trata de la primera investigación sobre factores de riesgo de anomalías congénitas con datos georreferenciados en el área de la cuenca Matanza Riachuelo, mostrando la factibilidad de efectuar este tipo de estudios con datos de la RENAC. Se han incluido diferentes variables socioeconómicas, reproductivas y habitacionales que permiten evaluar en forma más adecuada la población estudiada y se han utilizado diferentes metodologías para evaluar una misma hipótesis. Los resultados del presente estudio posiblemente podrán ser aplicados en futuras investigaciones de potencial riesgo teratogénico en el país.

En cuanto a las debilidades, el tamaño muestral pudo resultar limitado para identificar asociaciones específicas. El análisis de anomalías congénitas se realizó con el conjunto de los casos. Sin embargo las mismas son heterogéneas en sus causas. En el estudio se incluyeron para el análisis todas las industrias de un mismo rubro ubicadas en la cuenca. Sin embargo el tipo de agente y el grado de contaminación de cada establecimiento fabril aún pueden ser muy diferentes dentro del mismo rubro.

\section{Conclusión}

Si bien el resultado del presente estudio no ha demostrado asociación entre contaminantes ambientales y riesgo de anomalías congénitas, en cuanto a las políticas de control de la exposición a contaminantes por parte de la población, creemos que deben ser guiadas por el principio precautorio y no por el resultado de estudios individuales. El principio precautorio es un concepto que respalda la adopción de medidas preventivas ante la sospecha de que ciertos productos representen un riesgo grave para la salud pública, pero sin que se cuente todavía con pruebas científicas definitivas de tal riesgo ${ }^{22}$. Mientras que la prevención se aplica a causas conocidas, la precaución tiene mayor relevancia en el caso de factores de riesgo sospechados en escenarios complejos. En Argentina, la Ley Nacional del Ambiente (Ley 25.675) define al principio precautorio en su artículo cuatro: "Cuando haya peligro de daño grave o irreversible, la ausencia de información o certeza científicas no deberá utilizarse como razón para postergar la adopción de medidas eficaces, en función de los costos, para impedir la degradación del medio ambiente".

Limitaciones de responsabilidad: No hay conflicto de intereses. La investigación fue aprobada por los comités de ética de las instituciones participantes

Fuentes de apoyo: Este estudio fue parcialmente financiado por Salud Investiga del Ministerio de Salud de la Nación a través de un estudio multicéntrico. 


\section{Bibliografía}

1. DEIS, Estadísticas Vitales, Información Básica Año 2016. DEIS: Programa Nacional de Estadísticas de Salud. [Disponible en: www.deis.msal.gov.ar] [Consulta: 24-05-2018].

2. Observatorio Metropolitano [Disponible en http://www.observatorioamba.org/planes-y-proyectos/cuencas/cuencamatanza-riachuelo] [Consulta: 24-05-2018].

3. Autoridad de Cuenca Matanza- Riachuelo (ACUMAR), Eje ambiental [Disponible en http://www.acumar.gob.ar/ejeambiental] [Consulta: 24-05-2018].

4. Castilla EE, JS Lopez-Camelo, H Campana, and M Rittler. Epidemiological methods to assess the correlation between industrial contaminants and rates of congenital anomalies. Mutat Res, Dec 2001; 489(2-3): 123-45.

5. Groisman B, Bidondo MP, Barbero P, Gili JA, Liascovich R; RENAC Task Force.

RENAC: National Registry of Congenital Anomalies of Argentina. Arch Argent Pediatr. 2013 Dec;111(6):484-94

6. Segunda Encuesta nacional de factores de riesgo 2009 http://www.msal.gob.ar/images/stories/bes/graficos/0000000570cnt-2014-10_encuesta-nacional-factores-riesgo2011_informe-final.pdf [Consulta: 24-05-2018].

7. Castilla E, Lopez Camelo J, Paz J, Orioli I. Prevención primaria de los defectos congénitos. Ed. Fio Cruz 1996. Rio de Janeiro.

8. Pawluk MS, Campaña H, Gili JA, Comas B, Giménez LG, Villalba MI, Scala SC, Poletta FA, López Camelo JS. Adverse social determinants and risk for congenital anomalies.Arch Argent Pediatr. 2014 Jun;112(3):215-23.

9. Vrijheid M, Dolk H, Stone D, Abramsky L, Alberman E, Scott JE. Socioeconomic inequalities in risk of congenital anomaly. Arch Dis Child. 2000 May;82(5):349-52.

10. Silvestrin S, Silva CH, Hirakata VN, Goldani AA, Silveira PP, Goldani MZ. Maternal education level and low birth weight: a meta-analysis. J Pediatr (Rio J). 2013 Jul-Aug;89(4):339-45.

11. Geschwind SA, Stolwijk JA, Bracken M, et al. Risk of congenital malformations associated with proximity to hazardous waste sites. Am J Epidemiol 1992;135:1197- 1207.

12. Dolk $H$, Vrijheid $M$, Armstrong $B$, et al. Risk of congenital anomalies living near hazardous-waste landfill sites in Europe: the EUROHAZCON study. Lancet 1998;352:423-7.

13. Elliott $P$, Briggs $D$, Morris $S$, et al. Risk of adverse birth outcomes in populations living near landfill sites. BMJ 2001;323:363-8.

14. Dummer TJB, Dickinson HO, Parker L. Adverse pregnancy outcomes near landfill sites in Cumbria, Northwest England, 1950-1993. Arch Environ Health 2003;58:692-8.

15. Palmer SR, Dunstan FDJ, Fielder $\mathrm{H}$, et al. Risk of congenital anomalies following the opening of landfill sites. Environ Health Perspect 2005;113:1362-5.

16. Gilbreath S, Kass PH. Fetal and neonatal deaths and congenital anomalies associated with open dumpsites in Alaska Native villages. Int J Circumpolar Health 2006;65:133-47.

17. Baldo CC, Campaña H, Gili JA, Poletta FA, Lopez-Camelo JS. Anencephaly and Residence near Textile Industries: An Epidemiological Case-control Study in South America. Journal of Basic \& Applied Genetics, 2008;19 (1): 1-10. 
18. Sosniak WA, Kaye WE, Gomez TM. Data linkage to explore the risk of low birthweight associated with maternal proximity to hazardous waste sites from the National Priorities List. Arch Environ Health 1994;49:251-5.

19. Marshall EG, Gensburg LJ, Deres DA, et al. Maternal residential exposure to hazardous wastes and risk of central nervous system and musculoskeletal birth defects. Arch Environ Health 1997;52:416-25.

20. Boyle E, Johnson H, Kelly A, et al. Congenital anomalies and proximity to landfill sites. Ir Med J 2004;97:16-18.

21. Nieuwenhuijsen MJ, Dadvand P, Grellier J, Martinez D, Vrijheid M. Environmental risk factors of pregnancy outcomes: a summary of recent meta-analyses of epidemiological studies. Environ Health. 2013 Jan 15;12-6.

22. Martuzzi M. The precautionary principle: in action for public health. Occup Environ Med. 2007 Sep;64(9):569-70. 